\title{
GENERALIZATION OF A THEOREM OF KOROUS ON THE BOUNDS OF ORTHONORMAL POLYNOMIALS
}

\author{
DUNHAM JACKSON
}

1. Introduction. An elementary treatment of the convergence of series of orthogonal polynomials is greatly facilitated if the polynomials of the orthonormal set are known to be uniformly bounded on the domain of orthogonality, or on a part of it where convergence is to be proved. ${ }^{1}$ A demonstration due to $\mathrm{J}$. Korous ${ }^{2}$ shows in a few lines that the orthonormal polynomials corresponding to a weight function $\rho \sigma$ on a finite interval are thus bounded, if the polynomials for weight $\rho$ have the desired property, and if the factor $\sigma$ satisfies a Lipschitz condition and has a positive lower bound on the entire domain of orthogonality. The purpose of this note is to show that the argument of Korous can be extended so as to apply under fairly general conditions to orthogonal polynomials in two real variables on an algebraic curve $^{3}$ and in particular to orthogonal trigonometric sums, ${ }^{4}$ which can be regarded as orthogonal polynomials on a circle. ${ }^{5} \mathrm{~A}$ problem of the same category has been discussed by Peebles ${ }^{6}$ with less simple hypotheses on the factor $\sigma$.

In the case of trigonometric sums it is known in advance that the orthonormal functions for weight $\rho \equiv 1$, namely $(2 \pi)^{-1 / 2}, \pi^{-1 / 2} \cos k x$, $\pi^{-1 / 2} \sin k x, k=1,2, \cdots$, are uniformly bounded, and a theory of the convergence of developments in series of orthogonal trigonometric sums is opened up immediately. For other algebraic curves the question of the existence of a weight function which gives rise to a bounded system of orthonormal polynomials is one requiring separate investigation, and the answer to this question is known at present only in particular instances. ${ }^{7}$ When the existence of a single such

\footnotetext{
Presented to the Society April 12, 1941 under the title Generalization of a theorem of Korous; received by the editors November 17, 1941.

1 See, for example, D. Jackson, Series of orthogonal polynomials, Annals of Mathematics, (2), vol. 34 (1933), pp. 527-545; pp. 531-538.

2 See G. Szegö, Orthogonal Polynomials, American Mathematical Society Colloquium Publications, vol. 23, 1939, p. 157; D. Jackson, Fourier Series and Orthogonal Polynomials, Carus Mathematical Monographs, no. 6, 1941, pp. 205-208.

${ }^{3}$ See D. Jackson, Orthogonal polynomials on a plane curve, Duke Mathematical Journal, vol. 3 (1937), pp. 228-236. This paper will be cited by the letter A.

${ }^{4}$ See, for example, D. Jackson, Orthogonal trigonometric sums, Annals of Mathematics, (2), vol. 34 (1933), pp. 799-814. This paper will be cited as B.

5 A, p. 234.]

${ }^{6}$ G. H. Peebles, this Bulletin, abstract 45-5-219.

7 See, for example, D. Jackson, this Bulletin, abstract 45-5-192; Fulton Koehler,
} 
$\rho$ has been established for a specified domain of integration of the type to be discussed below, the conclusions of the present note lead to a wide generalization of the convergence theory for series of orthogonal polynomials associated with the curve in question.

2. Statement of the problem. Let $C$ be an algebraic curve or a portion of an algebraic curve, degenerate or nondegenerate, consisting of a single connected piece of finite extent or a finite number of such pieces. Let $C$ be of the $m$ th degree, in the sense that there is a polynomial $\Pi(x, y)$ of the $m$ th degree in $x$ and $y$, but no polynomial of lower degree, which vanishes identically on $C$, while each irreducible factor of $I$ vanishes on a portion of $C$ of positive length. The expression "connected pieces" will be understood to mean "pieces each of which is connected," not "pieces connected with each other." A single "piece," on the other hand, may be made up of parts corresponding to different irreducible factors of $\Pi$; it may be, for example, the perimeter of a square, or a set of line segments radiating from a common point. Let $\rho(x, y)$ be a non-negative weight function which is integrable over $C$ with respect to arc length, and for each irreducible factor of $\Pi$ is positive on a set of positive measure on the corresponding part of $C$.

Under these circumstances ${ }^{8}$ there is a set of polynomials in $x$ and $y$ orthonormal on $C$ with arc length as variable of integration and $\rho$ as weight function, which includes just $m$ polynomials of the $n$th degree for each value of $n \geqq m$, and $n+1$ polynomials of the $n$th degree for $n<m$. Let $p_{n i}(x, y), n=0,1,2, \cdots ; i=1,2, \cdots, m$, be such an orthonormal set, with the supplementary convention that when $n+1<m, p_{n i} \equiv 0$ for $i>n+1$, and also, on occasion, that $p_{-1, i} \equiv 0$ for $1 \leqq i \leqq m$. The value of $\int_{c} \rho p_{n i}^{2} d s$ then is in every case either 1 or 0 .

Let $\sigma(x, y)$ be a function defined and having a positive lower bound $h$ on $C$, and satisfying the condition that if $P_{1}$ and $P_{2}$ are any two points on a connected portion of $C$, with coordinates $\left(x_{1}, y_{1}\right)$ and $\left(x_{2}, y_{2}\right)$,

$$
\left|\sigma\left(x_{2}, y_{2}\right)-\sigma\left(x_{1}, y_{1}\right)\right| \leqq \lambda \Delta s,
$$

where $\lambda$ is constant (for simplicity of notation, a single constant for the whole of $C$ ), and $\Delta s$ is the distance from $P_{1}$ to $P_{2}$ along $C$, or the shortest such distance, if $P_{1}$ and $P_{2}$ are connected by $C$ in more than one way.

Systems of orthogonal polynomials on certain algebraic curves, this Bulletin, vol. 46 (1940), pp. 345-351.

${ }^{8}$ See [A, pp. 232-234]. The present hypotheses are somewhat more general than those which were explicitly formulated in the earlier paper, but no essential change is required in the reasoning which leads to recognition of the form of the orthonormal system. 
Let $q_{n i}(x, y), n=0,1,2, \cdots ; i=1,2, \cdots, n+1$ for $n<m, i=1,2$, $\cdots, m$ for $n \geqq m$, be a set of orthonormal polynomials on $C$ with $\rho \sigma$ as weight function; there is no occasion to introduce identically vanishing $q$ 's.

The question at issue is that of inferring boundedness of the polynomials $q_{n i}$ from that of the polynomials $p_{n i}$. It will be found that such an inference is possible, under conditions to be specified.

3. Proof of the theorem for an arbitrary algebraic curve. Let

$$
K_{n}(x, y, u, v)=\sum_{k=0}^{n} \sum_{i=1}^{m} p_{k i}(x, y) p_{k i}(u, v) .
$$

Then any one of the polynomials $q_{n j}(x, y)$, as a polynomial of the $n$th degree, has on $C$ the representation

$$
\begin{aligned}
q_{n j}(x, y)= & \int_{C} \rho(u, v) K_{n}(x, y, u, v) q_{n j}(u, v) d s \\
= & \sum_{i=1}^{m} p_{n i}(x, y) \int_{C} \rho(u, v) p_{n i}(u, v) q_{n j}(u, v) d s \\
& +\int_{C} \rho(u, v) K_{n-1}(x, y, u, v) q_{n j}(u, v) d s
\end{aligned}
$$

the integration is extended over the curve $C$ in the $(u, v)$-plane. By application of Schwarz's inequality when $p_{n i} \neq 0$,

$$
\begin{aligned}
{\left[\int_{C} \rho p_{n i} q_{n j} d s\right]^{2} } & \leqq \int_{C} \rho p_{n i}^{2} d s \int_{C} \rho q_{n j}^{2} d s=\int_{C} \rho q_{n j}^{2} d s \\
& \leqq \frac{1}{h} \int_{C} \rho \sigma q_{n j}^{2} d s=\frac{1}{h},
\end{aligned}
$$

the $p$ 's being normalized for weight $\rho$ and the $q$ 's for weight $\rho \sigma$. It remains to examine in some detail the integral involving $K_{n-1}$. Let this integral be denoted by $I(x, y)$ or $I$.

The sum $K_{n-1}(x, y, u, v)$ has alternative representations of the form ${ }^{9}$

(3) $K_{n-1}=\frac{1}{u-x} \sum_{i=1}^{m} \sum_{l=1}^{m} c_{i l}\left[p_{n i}(u, v) p_{n-1, l}(x, y)-p_{n-1, l}(u, v) p_{n i}(x, y)\right]$,

(4) $K_{n-1}=\frac{1}{v-y} \sum_{i=1}^{m} \sum_{l=1}^{m} d_{i l}\left[p_{n i}(u, v) p_{n-1, l}(x, y)-p_{n-1, l}(u, v) p_{n i}(x, y)\right]$,

? [A, pp. 235-236. $]$ 
as special cases of a more general expression having an arbitrary linear combination of $u-x$ and $v-y$ in the denominator. If $G$ is a number such that $|x| \leqq G,|y| \leqq G$ on $C$, then

$$
\left|c_{i l}\right| \leqq G, \quad\left|d_{i l}\right| \leqq G
$$

for all values of the subscripts. For

$$
c_{i l}=\int_{C} \rho u p_{n i} p_{n-1, l} d s,
$$

and by another application of Schwarz's inequality

$$
c_{i l}^{2} \leqq \int_{C} \rho u^{2} p_{n i}^{2} d s \int_{C} \rho p_{n-1, l}^{2} d s \leqq G^{2} \int_{C} \rho p_{n i}^{2} d s=G^{2}
$$

a similar calculation applies to $d_{i l}$.

Since $K_{n-1}(x, y, u, v)$ is for fixed $(x, y)$ a polynomial in $(u, v)$ of degree lower than the $n$ th, it follows from the property of orthogonality of $q_{n j}$ that

$$
\int_{C} \rho(u, v) \sigma(u, v) K_{n-1}(x, y, u, v) q_{n j}(u, v) d s=0 .
$$

Consequently $\sigma(x, y) I$ can be represented in the form

$$
\sigma(x, y) I=\int_{C} \rho(u, v)[\sigma(x, y)-\sigma(u, v)] K_{n-1}(x, y, u, v) q_{n j}(u, v) d s .
$$

Let $C_{1}$ be a portion of $C$ forming a closed point set, consisting of a finite number of connected pieces, and containing no singular point, that is, neither a singular point of any nondegenerate component of $C$ nor any point common to two such components. Then each connected part of $C_{1}$ is a straight line segment, a smooth arc, or a smooth closed loop.

The essential ideas of the argument are illustrated with a simpler initial formulation, and in a form adequate for some of the most interesting applications, if it is assumed that $C$ itself has the characteristics ascribed to $C_{1}$, and that $C_{1}$ is the whole of $C_{\text {; }}$ the method is however of wider applicability as indicated. On the other hand, the reasoning could be made still more general, at the expense of some further attention to details, but the usefulness of the additional generality would not be so immediately apparent. The need for explicit consideration of certain wholly elementary items of detail arises from the fact that for fixed $(x, y)$ either of the denominators $u-x, v-y$ 
in the expressions for $K_{n-1}$ may vanish not only at the point $u=x$, $v=y$, but also at a number of other points of the curve.

At any point of $C_{1}$ the derivatives $d y / d x$ and $d x / d y$ have definite values, or else one of them has the value 0 . Except on straight line segments, $d y / d x$ can take on the values \pm 1 at only a finite number of points. The locus $C_{1}$ is made up of a finite number of arcs or segments, on any one of which $|d y / d x|$ is everywhere greater than or equal to 1 or else everywhere less than or equal to 1 . Any such arc $C^{\prime}$ (the word $\operatorname{arc}$ being regarded now and henceforth as applicable in particular to a line segment) is included in or coextensive with an arc $C^{\prime \prime}$, also belonging to $C$, on which $|d y / d x|$ is everywhere greater than or equal to $1 / 2$, if it is greater than or equal to 1 on $C^{\prime}$, or everywhere less than or equal to 2 , if it is less than or equal to 1 on $C^{\prime}$, and such that each end of $C^{\prime}$, if not a terminal point of $C$, is an interior point of $C^{\prime \prime}$. Let a definite arc $C^{\prime \prime}$ be associated in this way with each $C^{\prime}$. The distance from any point of an arc $C^{\prime}$ to any point of $C$ not belonging to the corresponding arc $C^{\prime \prime}$ has a positive lower bound $\delta$ for the whole of the locus $C_{1}$ which constitutes the aggregate of the arcs $C^{\prime}$. It is to be noted further that $|d s / d x| \leqq 5^{1 / 2}$ on an arc $C^{\prime \prime}$ where $|d y / d x| \leqq 2$, and $|d s / d y| \leqq 5^{1 / 2}$ on the other type of arc $C^{\prime \prime}$.

Let $P$, with coordinates $(x, y)$, be an arbitrary point of $C_{1}$. Let $C_{P}^{\prime}$ be the arc $C^{\prime}$ to which $P$ belongs, or, if $P$ is a common end point of two such arcs, let $C_{P}^{\prime}$ be either of them. Let $K$ be the corresponding arc $C^{\prime \prime}$. Let the rest of $C$ be subdivided into two parts $K^{\prime}, K^{\prime \prime}$, so that if $P^{\prime}$, with coordinates $(u, v)$, is any point of $K^{\prime}$, the line $P P^{\prime}$ makes an angle not greater than $\pi / 4$ with the $x$-axis, and if $P^{\prime}$ is on $K^{\prime \prime}, P P^{\prime}$ makes an angle less than $\pi / 4$ with the $y$-axis. With the value of $\delta$ defined in the preceding paragraph, $|u-x| \geqq \delta / 2^{1 / 2}$ for any point $P^{\prime}$ on $K^{\prime}$, and $|v-y| \geqq \delta / 2^{1 / 2}$ if $P^{\prime}$ is on $K^{\prime \prime}$.

Let it be supposed for definiteness that $C_{P}{ }^{\prime}$ is an arc of slope numerically less than or equal to 1 ; the alternative case would be treated in the same way, with the obvious interchanges of variables. For integration in (5), the point $(x, y)$ being regarded as fixed, let $K_{n-1}(x, y, u, v)$ be represented by $(3)$ when $(u, v)$ is on $K$ or $K^{\prime}$, and by (4) when $(u, v)$ is on $K^{\prime \prime}$.

On $K$, since $\Delta s \leqq 5^{1 / 2}|\Delta x|$,

$$
|\sigma(x, y)-\sigma(u, v)| \leqq 5^{1 / 2} \lambda|u-x| \text {. }
$$

Let $\mu$ be an upper bound for $\sigma$ on the whole of $C$. Then on $K^{\prime}$,

$$
|[\sigma(x, y)-\sigma(u, v)] /(u-x)| \leqq 2^{3 / 2} \mu / \delta,
$$

and there is a similar inequality on $K^{\prime \prime}$ with $u-x$ replaced by $v-y$. 
By application of Schwarz's inequality, as in (2),

$$
\int_{C} \rho(u, v)\left|p_{\alpha \beta}(u, v) q_{n j}(u, v)\right| d s \leqq h^{-1 / 2},
$$

and the same upper bound is valid if the integration is extended only over $K, K^{\prime}$, or $K^{\prime \prime}$.

Let it be supposed that $\left|p_{\alpha \beta}(x, y)\right| \leqq H$ on a point set $C_{2}$ contained in $C_{1}$, uniformly for all values of the subscripts $\alpha$ and $\beta$. (In particular, $C_{2}$ may be the whole of $C_{1}$.) Then, by combination of the inequalities that have been obtained,

$$
\sigma(x, y)|I(x, y)| \leqq\left(5^{1 / 2} \lambda+2^{5 / 2} \mu / \delta\right) 2 m^{2} G h^{-1 / 2} H
$$

if $(x, y)$ is on $C_{2}$. (It is readily seen that the coefficient $2^{5 / 2}=2 \cdot 2^{3 / 2}$ could be replaced by $2^{3 / 2}$, but the difference is immaterial for the purpose in hand.) The absolute value of the sum in the third member of (1) does not exceed $m h^{-1 / 2} H$. Consequently, for $(x, y)$ on $C_{2}$,

$$
\left|q_{n j}(x, y)\right| \leqq H\left[m h^{-1 / 2}+\left(5^{1 / 2} \lambda+2^{5 / 2} \mu / \delta\right) 2 m^{2} G h^{-3 / 2}\right] .
$$

Since the right-hand member is independent of $x, y, j$, and $n$, it follows that if the p's are uniformly bounded on $C_{2}$, the same is true of the $q$ 's.

4. Orthogonal trigonometric sums. In a particular case the results can be interpreted as relating to orthogonal trigonometric sums, the unit circle or a finite set of arcs on the unit circle being taken for $C$ and $C_{1}$. With $x=\cos \theta, y=\sin \theta$, the polynomials $p_{n i}(x, y), q_{n i}(x, y)$ are trigonometric sums in $\theta$. The weight function $\rho(x, y)$ and the factor $\sigma(x, y)$ will be represented alternatively by $\rho(\theta)$ and $\sigma(\theta)$. As far as the definition of the orthonormal system is concerned, the case of a domain of orthogonality consisting of detached intervals in a period is of course equivalent to that of a domain consisting of the entire period, with a weight function which vanishes identically outside the intervals in question. The conclusion can be stated as follows:

Let $u_{0}(\theta), u_{1}(\theta), v_{1}(\theta), \cdots$ constitute a set of orthonormal trigonometric sums with respect to a weight function $\rho(\theta)$ on a domain $D_{1}$ consisting of a period interval, regarded as closed, or of a finite number of closed intervals contained in a period; let $\sigma(\theta)$ have a positive lower bound on $D_{1}$, and satisfy a condition of the form

$$
\left|\sigma\left(\theta_{2}\right)-\sigma\left(\theta_{1}\right)\right| \leqq \lambda\left|\theta_{2}-\theta_{1}\right|
$$

on each interval of $D_{1}$, taking on the same value at both ends of the period interval if these end points belong to $D_{1} ;$ and let $U_{0}(\theta), U_{1}(\theta), V_{1}(\theta), \ldots$ 
be the orthonormal trigonometric sums on $D_{1}$ for weight $\rho(\theta) \sigma(\theta)$; if the $u$ 's and $v$ 's are uniformly bounded on a point set $D_{2}$ contained in $D_{1}$, the same is true of the U's and V's.

For this case the proof admits a materially simpler formulation than when geometric configurations are contemplated having the degree of generality previously considered. The details relating to the loci $C^{\prime}, C^{\prime \prime}, K, K^{\prime}, K^{\prime \prime}$, can be dispensed with for the most part; with $\theta$ replacing the pair of coordinates $(x, y)$, and $\phi$ replacing the pair $(u, v)$, it is sufficient, for any particular value of $\theta$, to consider separately the intervals $(\theta-\pi / 2, \theta+\pi / 2)$ and $(\theta+\pi / 2, \theta+3 \pi / 2)$, and in the integral corresponding to the right-hand member of (5) to represent $K_{n-1}(\theta, \phi)$ in the former interval by an expression with denominator $\sin (\theta-\phi)$, and in the latter interval by an alternative expression with $1-\cos (\theta-\phi)$ in the denominator. ${ }^{10}$

The University of Minnesota

10 [B, pp. 808-809.]

\section{APPROXIMATION OF CONTINUOUS FUNCTIONS BY MEANS OF LACUNARY POLYNOMIALS}

\section{BERNARD DIMSDALE}

1. Introduction. All rational integral polynomials are linear combinations of members of the complete set of powers whose exponents are the non-negative integers. If certain members of this set are deleted, the linear combinations formed from the resulting set are, in the strict sense of the term, "lacunary polynomials." In a large part of this paper, however, methods of reasoning designed for the treatment of such polynomials are applicable to combinations from much more general sets of powers whose exponents are non-negative but not in general integral. The term "polynomial in $x^{\mu}$ of degree $\mu_{n}$ " will be applied to combinations from the set $1, x^{\mu_{1}}, x^{\mu_{2}}, \cdots$ where $\mu_{1}, \mu_{2}, \cdots$ form an arbitrarily preassigned set of real numbers such that $0<\mu_{1}<\mu_{2}<\cdots$, and $\mu_{n}$ is the largest exponent.

This paper started out as an investigation of lacunary orthogonal polynomials, and although this aspect of it became subordinate to the

Presented to the Society, September 7, 1939 under the title Degree of approximation by linear combinations of powers; received by the editors March 20,1941, and, in revised form, October 29, 1941. 